\title{
Capacity of Cooperative Spatial Modulation (CSM) System with Optimum Relay Selection
}

\author{
Jamal A. Hassan (D) and Samah A. Mustafa \\ Electrical Engineering Department, University of Salahaddin, Erbil, Iraq \\ Correspondence should be addressed to Jamal A. Hassan; jamal.hassan@su.edu.krd
}

Received 12 February 2020; Revised 23 June 2020; Accepted 7 July 2020; Published 24 July 2020

Academic Editor: Tuan M. Nguyen

Copyright (C) 2020 Jamal A. Hassan and Samah A. Mustafa. This is an open access article distributed under the Creative Commons Attribution License, which permits unrestricted use, distribution, and reproduction in any medium, provided the original work is properly cited.

\begin{abstract}
In this work, the capacity of the cooperative spatial modulation (CSM) system is analysed. Relay-aided transmission is considered to improve the coverage and the reliability using decode and forward scheme. Due to the nonclosed form of the spatial modulation capacity function, its upper and lower bounds are derived. The lower bound of the capacity is exploited to formulate the optimal relay selection problem to maximize the channel utilization, where the optimal relay selection algorithm (ORSA) and modified maximum harmonic mean (MMHM) are proposed to select single or multiple relays. A comparison study in terms of the performance and the computational complexity is presented for the evaluation purpose. Simulation results demonstrate that the proposed algorithm significantly improves the capacity of CSM compared to other most popular relay selection strategies.
\end{abstract}

\section{Introduction}

The emergence of multiple-input multiple-output (MIMO), massive MIMO, and spatial modulation in the last decade demonstrates the importance of spatial diversity for improving system performance. However, some practical issues are related to system complexity and cost. Special attention to interchannel interference and interantenna synchronization is required due to the need for implementation of multiple radio frequency (RF) chains (one for each antenna) [1]. Spatial modulation (SM) has been proposed for MIMO communication with a single RF chain at the transmitter in which the transmitting antenna index is used as a source of information. According to the transmitted sequence, a symbol of the constellation is selected and transmitted via a selected antenna out of a set. Using a multiple-antenna system will provide diversity, multiplexing, and array gain $[2,3]$. The multiple antenna diversity is a type of spatial diversity, which, in contrast to time diversity, has the advantage of achieving gain without reducing the effective data transmission rate [4]. In addition, the multiplexing gain that is achieved by spatial modulation increases spectral efficiency by where the number of the transmitting antennas increased, in compari- son with the typical method of transmission with one antenna. In this method, in addition to estimating the transmitted symbols, the receiver also estimates the transmitted antenna index, which contains a portion of the transmitted information. Furthermore, the benefit of this method in comparison with other methods, such as vertical Bell Lab layered space-time (V-BLAST) and space-time coding (STC), is the higher spectral efficiency [5].

In [6], based on the proposed transmission method, namely, the information-guided channel hopping (IGCH) scheme, the capacity of multiple transmitter antennas is studied. In this scheme, the independent character of multichannel can be used as an additional information transmitting channel. The analysis results prove that the capacity behavior of this scheme is better than that of the space-time block coding for more than two transmit antennas.

In [7], to maximize the instantaneous capacity of SM for the spatial domain, an iterative algorithm is proposed, in which the optimal probability of activating each transmit antenna is obtained. There is a trade-off between design freedom and the diversity gain achieved; therefore, obtaining optimal spatial domain design is necessary. However, the computational complexity of the iterative method is very 
high. In [8], the authors derived lower and upper bounds of generalized spatial modulation's (GSM's) capacity, in which multiple antennas are used at both transmitter and receiver with more than one RF chain. The authors in [9] derived a closed form expression for SM capacity with only two transmit and single receiver antennas based on the hypergeometric series. An increasing number of the antenna in the transmitter dramatically increase the complexity of mathematical calculation.

In $5 \mathrm{G}$ mobile communication systems, millimeter-wave wireless communication is a key enabling technology where the coverage with a very high data rate is limited. A cooperative framework in the new generation of wireless communication via relaying the transmit signal is a promising approach to extend the coverage range, combat the channel fading, and leverage the channel utilization. The research community has explored the relay selection problem to achieve the goals. In [10], random relay selection (RRS) is considered with no computational overhead; however, this relay selection scheme cannot provide optimal performance. Maximum harmonic mean relay selection (MHM-RS) is a method in which the relay that corresponds to the highest harmonic mean of the source-relay link (SRL) and relaydestination link (RDL) conditions is selected [11]. Several metrics are presented in [12]. These criteria are based on the quality of the communication links, and the transmission powers of the relay and source are ignored. Partial relay selection in the orthogonal frequency division multiplexing with index modulation (OFDM-IM) system is proposed in [13] to evaluate average symbol error probability. The relay is selected based on the largest channel gain in the first hop only. However, the criteria considering both transmission phase provide better performance. In our previous published work [14], we presented efficient partner selection metrics and conclude that employing multiple relays in the cooperative process improves the error performance and the capacity even with interference impacts.

Cooperative spatial modulation (CSM) exploits the advantages of SM and cooperative communication. In the CSM network, there are multiple links between the source and each relay; thus, the criteria for relay selection must be modified based on the CSM specification. CSM based on multiple DF relays was studied in [15]. The upper bound of the pairwise error probability (PEP) is analysed to investigate the performance of the system; however, no optimum relay is selected, and all the existing relays are used in the cooperative process to improve the performance of the system at the expense of high power consumption and additional overheads. In [16], there is CSM with AF relay scheme in which the relay is selected based on link quality in the second transmission phase. The source applies space shift keying (SSK) as a special form of SM where only the active antenna index conveys the information. Thus, the throughput of cooperative SSK is limited. In [17], the system performance in terms of symbol error probability under various conditions, such as half-duplex (HD), full-duplex (FD), existence, and absence of a direct link between the source and the destination, is studied. The residual interference between relay transmit and receive antennas is considered. Only a capacity equation that is based on the signal space is obtained, and no further analysis is conducted. The authors in [18] proposed a new CSM system where all the nodes are equipped with multiple transmit and/or receive antennas. The system recorded better error performance than classical cooperative systems; however, installing multiple antennas on all the nodes might not be possible. For the CSM network in [19], a relay is selected based on the quality of the output signal from the maximum likelihood (ML) detector in the receiver. However, a predefined relay selection scheme is more realistic in terms of the network design, regardless of the output of the detector. A quadrature CSM network with DF relaying scheme is proposed in [20]. To improve spectral efficiency, a source jointly selects the active antenna, a single relay, and a constellation symbol based on the information bits. Ignoring the quality of the links in the relay selection cannot help the system to improve its performance. In addition, the computational complexity of the joint selection of the mentioned factors is quite high and causes a significant delay in the transmission process.

According to the earlier description and the gains that can be harvested, CSM networks based on the DF relaying scheme are considered in this paper in which only the source has multiple antennas. It is assumed that the direct link between the source and the destination does not exist, and a single relay at least is necessary for the communication process. The work is aimed at maximizing the channel utilization through a relay selection policy.

The main contribution of this paper includes the following aspects. First is the theoretical analysis of the SM capacity and derivation of a lower and an upper bound of the capacity to be used in the study and avoidance of the complexity of dealing with the nonclosed form of SM capacity. Next, to exploit the cooperating relays wisely and achieve a satisfying gain, new relay selection criteria based on the channel quality are proposed in addition to a modified maximum harmonic mean (MMHM) to be used in the CSM network as a lowcomplexity suboptimal relay selection scheme. As another contribution of the work, an optimal relay selection algorithm (ORSA) is presented with slightly higher computational complexity than MMHM. Numerical simulations have been presented to evaluate the performance of the CSM system using these selection policies and compare it with that using an exhaustive search algorithm to define a cooperative relay.

The remainder of this paper is organized into four sections. Section 2 presents the system model and the capacity calculation of SM. Relay selection problems in CSM are presented in Section 3. Then, the numerical results are discussed in Section 4. Finally, the conclusions of the work are presented in Section 5.

\section{System Model}

A cooperative spatial modulation CSM network based on the DF relay scheme is considered, as illustrated in Figure 1. The source has $N$ antennas and transmits the information signal using a spatial modulation (SM) scheme in the first phase. We assume that a direct link between the source and the 


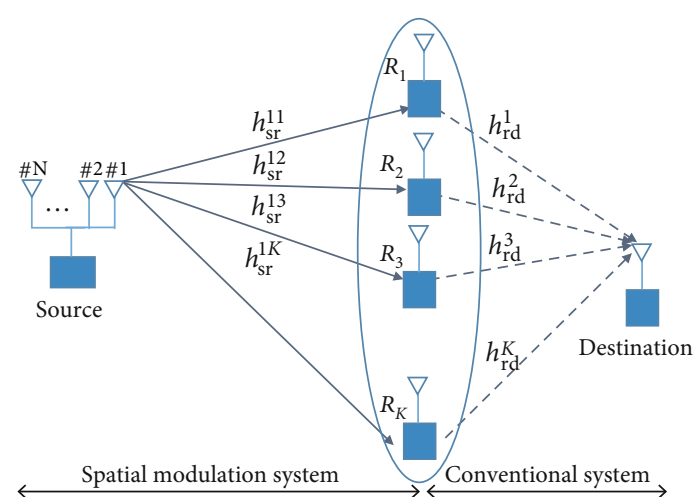

FIgURE 1: Cooperative network with spatial modulation at the source.

destination does not exist. Several surrounding relays, each with a single antenna, will receive the signal. The relay who decodes the original signal correctly is the candidate to forward the signal to the destination in the DF scheme.

The CSM system is a dual-hop cooperative system. In the first hop, the SM system is used, while the conventional modulation system is used in the next hop. The capacity of CSM with the $j$ th selected relay $\left(C_{\mathrm{csm}}^{j}\right)$ based on the DF scheme is obtained as [21]

$$
C_{\mathrm{csm}}^{j}=\min \left\{C_{\mathrm{sr}}^{j}, C_{\mathrm{rd}}^{j}\right\}
$$

where $C_{\mathrm{sr}}^{j}$ and $C_{\mathrm{rd}}^{j}$ are the capacity of the source-to-jth relay and the capacity of the $j$ th relay-to-destination, respectively. To facilitate the upcoming discussions, the main notations that are used in the paper are listed in Table 1 . The capacity of each transmission phase is evaluated separately.

2.1. SM Capacity of the Source-Relay Links. In the first transmission phase, SM transmits $\log _{2}(M N)$ bits, of which $\log _{2}$ $(M)$ bits are used to select a constellation symbol and $\log _{2}(N)$ bits are used to set the index of the active antenna to transmit the symbol. If the $i$ th transmission antenna is defined to transmit the $q$ th symbol, the received signal at the $j$ th relay $\left(y_{\mathrm{rj}}\right)$ is represented as

$$
y_{\mathrm{rj}}=\sqrt{P_{\mathrm{s}}} \mathbf{h}_{\mathrm{sr}}^{i j} \mathbf{x}+n_{\mathrm{rj}}
$$

where $\mathbf{x}$ is an $N$-dimensional symbol vector and symbol $x_{q}$ is the $q$ th element of $\mathbf{x}$, which represents the $q$ th element of the $M$-ary constellation state:

$$
\mathbf{x}=\left[\begin{array}{llllll}
0 & 0 & \cdots & x_{q} & \cdots & 0
\end{array}\right]^{T}, \quad \text { for } q=1, \cdots, M
$$

$\mathbf{h}_{\mathrm{sr}}^{i j}$ is the fading channel coefficients of the link between the $i$ th transmission antenna and the $j$ th relay. $P_{\mathrm{s}}$ is the
TABLE 1: System parameters.

\begin{tabular}{lc}
\hline Symbol & Quantity \\
\hline$h_{\mathrm{sr}}^{i j}$ & $\begin{array}{r}\text { Channel coefficient between the } i \text { th antenna } \\
\text { and the } j \text { th relay }\end{array}$ \\
$\begin{array}{c}h_{\mathrm{rd}}^{j} \\
N\end{array}$ & $\begin{array}{c}\text { Channel coefficient between the } j \text { th relay and } \\
\text { the destination }\end{array}$ \\
$M$ & Number of antennas at the source \\
$P_{\mathrm{s}}, P_{\mathrm{r}}, P_{\text {tot }}$ & Size of constellation \\
$C_{\mathrm{csm}}^{j}$ & The capacity of CSM with the selection of the $j$ th relay \\
$C_{\mathrm{sr}}^{j}$ & The capacity of the source to the $j$ th relay \\
$C_{\mathrm{rd}}^{j}$ & The capacity of the $j$ th relay to the destination \\
$K$ & Number of available relays \\
$\sigma_{n}^{2}$ & Noise power
\end{tabular}

source transmission power, and $n_{\mathrm{rj}}$ is an additive white complex Gaussian noise with zero mean and variance of $\sigma_{n}^{2}$.

At the source SM scheme, transmission is simultaneously conducted for two data streams: one data stream is mapped to the index of the selected transmit antenna $\left(x_{\text {ant }}\right)$, and the other data stream is conventionally encoded and transmitted by the selected antenna $(x)$. There will be two independent input signal spaces for the symbol and antenna symbol, which are denoted as $X$ and $X_{\text {ant }}$, in addition to the output signal in the $j$ th relay space $Y_{j}$. The mutual information between the input and output of the signal space is [22]

$$
I\left(X, X_{\mathrm{ant}} ; Y_{j}\right)=I\left(X ; Y_{j} \mid X_{\mathrm{ant}}\right)+I\left(X_{\mathrm{ant}} ; Y_{j}\right) .
$$

Consider a random antenna selection probability of $1 / N$. The capacity of the signal space $C_{1, j}$ is calculated as

$$
C_{1, j}=\max _{p(x)} I\left(X ; Y_{j} \mid X_{\text {ant }}\right)=\frac{1}{N} \sum_{i=1}^{N} \log _{2}\left(1+\frac{\left|\mathbf{h}_{\mathrm{sr}}^{i j}\right|^{2} P_{s}}{\sigma_{n}^{2}}\right)
$$

$p(x)$ is the probability density function of the random transmitted symbol vector $x$.

The capacity of the antenna space $C_{2, j}$ is calculated as

$$
\begin{aligned}
C_{2, j}= & \frac{1}{N} \sum_{i=1}^{N} \int\left\{p\left(y_{\mathrm{rj}} \mid x_{\mathrm{ant}}=i^{(\uparrow)}\right) \log _{2} p\left(y_{\mathrm{rj}} \mid x_{\mathrm{ant}}=i^{(\uparrow)}\right)\right. \\
& \left.\cdot \log _{2} p\left(y_{\mathrm{rj}} \mid x_{\mathrm{ant}}=i^{(\uparrow)}\right)-p\left(y_{\mathrm{rj}} \mid x_{\mathrm{ant}}=i^{(\uparrow)}\right) \log _{2} p\left(y_{j}\right)\right\} d y_{\mathrm{rj}},
\end{aligned}
$$


where

$$
\begin{aligned}
p\left(y_{\mathrm{rj}} \mid x_{\mathrm{ant}}=i^{(\uparrow)}\right) & =\frac{1}{\pi \sigma_{i j}^{2}} \exp \left(-\frac{\left|y_{j}\right|^{2}}{\sigma_{i j}^{2}}\right), \\
p\left(y_{\mathrm{rj}}\right) & =\sum_{i=1}^{N} p\left(y_{\mathrm{rj}} \mid x_{\mathrm{ant}}=i^{(\uparrow)}\right) p\left(x_{\mathrm{ant}}=i^{(\uparrow)}\right) \\
& =\frac{1}{N} \sum_{i=1}^{N} \frac{1}{\pi \sigma_{i j}^{2}} \exp \left(-\frac{\left|y_{\mathrm{rj}}\right|^{2}}{\sigma_{i j}^{2}}\right),
\end{aligned}
$$

in which $\sigma_{i j}^{2}=\left|h_{\mathrm{s}}^{i j}\right|^{2} P_{\mathrm{s}}+\sigma_{n}^{2}$ is the variance of the received signal through the $i$ th antenna. $i{ }^{(\uparrow)}$ denotes that the $i$ th antenna is active and equivalent to the transmitting symbol $x_{\text {ant }}$. The first term of the right-hand side of (6) can be expressed as

$$
\begin{aligned}
& \frac{1}{N} \sum_{i=1}^{N} \int_{y_{j}} p\left(y_{\mathrm{rj}} \mid x_{\mathrm{ant}}=i^{(\uparrow)}\right) \log _{2} p\left(y_{\mathrm{rj}} \mid x_{\mathrm{ant}}=i^{(\uparrow)}\right) d y_{\mathrm{rj}} \\
& \quad=-\frac{1}{N} \sum_{i=1}^{N} \log _{2}\left(\pi \sigma_{i j}^{2} e\right) .
\end{aligned}
$$

The second term of the right-hand side of (6) can be expressed as

$$
\begin{aligned}
\Lambda\left(\sigma_{i j}^{2}\right) & =-\frac{1}{N} \sum_{i=1}^{N} \int_{y} p\left(y_{j} \mid x_{\mathrm{ant}}=i^{(\uparrow)}\right) \log _{2}\left(p\left(y_{\mathrm{rj}}\right)\right) d y_{\mathrm{rj}} \\
& =-\frac{1}{N} \sum_{i=1}^{N} \int_{0}^{\infty} \frac{1}{\sigma_{i j}^{2}} e^{-\left(r / \sigma_{i j}^{2}\right)} \log _{2}\left(\frac{1}{N} \sum_{k=1}^{N} \frac{1}{\pi \sigma_{k j}^{2}} e^{-\left(r / \sigma_{k j}^{2}\right)}\right) d r \\
& =-\frac{1}{N} \sum_{i=1}^{N} \int_{0}^{\infty} h_{i j}(r) d r .
\end{aligned}
$$

From (8) and (9), the capacity $C_{2, j}$ can be obtained as

$$
C_{2, j}=-\frac{1}{N} \sum_{i=1}^{N} \log _{2}\left(\pi \sigma_{i j}^{2} e\right)-\frac{1}{N} \sum_{i=1}^{N} \int_{0}^{\infty} h_{i j}(r) d r,
$$

in which

$$
h_{i j}(r)=\frac{1}{\sigma_{i j}^{2}} e^{-\left(r / \sigma_{i j}^{2}\right)} \log _{2}\left(\frac{1}{N} \sum_{k=1}^{N} \frac{1}{\pi \sigma_{k j}^{2}} e^{-\left(r / \sigma_{k j}^{2}\right)}\right) .
$$

Now, from (4), (5), and (10), the capacity of the spatial modulation is given by

$$
C_{\mathrm{sr}}^{j}=C_{1 j}+C_{2 j}=-\log _{2}\left(\pi \sigma_{n}^{2} e\right)-\frac{1}{N} \sum_{i=1}^{N} \int_{0}^{\infty} h_{i j}(r) d r .
$$

The analytical solution to obtain the capacity of $\mathrm{SM} C_{\mathrm{sr}}^{j}$ is difficult because of its nonclosed form. Since the statistical analysis is not sufficient for understanding the properties of SM, to facilitate understanding and for practical applications, such as resource allocation, we will define upper and lower bounds of $C_{\mathrm{sr}}^{j}$.

2.1.1. Lower Bound of the SM Capacity. The term $\left(1 / \sigma_{i j}^{2}\right)$ $e^{-\left(r / \sigma_{i j}^{2}\right)}$ in (9) is an exponential distribution, and the second term in the right-hand side of (12) can be expressed as the expectation of random variables $\Lambda\left(\boldsymbol{\sigma}_{\mathbf{j}}\right)$, in which $\boldsymbol{\sigma}_{\mathbf{j}}=$ $\left[\sigma_{1 j}^{2} \sigma_{2 j}^{2} \cdots \sigma_{N j}^{2}\right]^{T}$ :

$$
\Lambda\left(\boldsymbol{\sigma}_{\mathbf{j}}\right)=-\frac{1}{N} \sum_{i=1}^{N} E_{i j}\left\{\log _{2}\left(\frac{1}{N} \sum_{k=1}^{N} \frac{1}{\pi \sigma_{k j}^{2}} e^{-\left(r / \sigma_{k j}^{2}\right)}\right)\right\},
$$

where $E_{i j}\{\cdot\}$ denotes the $i$ th antenna and $j$ th relay expectation that corresponds to the statistical distribution with pdf of $f_{i j}(r) \triangleq\left(1 / \sigma_{i j}^{2}\right) e^{-\left(r / \sigma_{i j}^{2}\right)}$. On the other hand, according to Jensen's inequality, the convex function in (13) can be expressed as

$$
-\frac{1}{N} \sum_{i=1}^{N} \log _{2}\left(E_{i j}\left\{\frac{1}{N} \sum_{k=1}^{N} \frac{1}{\pi \sigma_{k j}^{2}} e^{-\left(r / \sigma_{k j}^{2}\right)}\right\}\right) \leq \Lambda\left(\boldsymbol{\sigma}_{\mathbf{j}}\right) .
$$

A lower bound for $\Lambda\left(\boldsymbol{\sigma}_{\mathbf{j}}\right)$ can be defined as

$$
L\left(\boldsymbol{\sigma}_{\mathbf{j}}\right)=-\frac{1}{N} \sum_{i=1}^{N} \log _{2}\left(E_{i j}\left\{\frac{1}{N} \sum_{k=1}^{N} \frac{1}{\pi \sigma_{k j}^{2}} e^{-\left(r / \sigma_{k j}^{2}\right)}\right\}\right) .
$$

For computing $L\left(\boldsymbol{\sigma}_{\mathbf{j}}\right)$, we obtain

$$
\begin{aligned}
E_{i j}\left\{\frac{1}{N} \sum_{k=1}^{N} \frac{1}{\pi \sigma_{k j}^{2}} e^{-\left(r / \sigma_{k j}^{2}\right)}\right\} & =\frac{1}{\pi N} \int_{0}^{\infty} f_{i j}(r) \sum_{j=1}^{N} \frac{1}{\sigma_{k j}^{2}} e^{-\left(r / \sigma_{k j}^{2}\right)} d r \\
& =\frac{1}{\pi N} \sum_{k=1}^{N} \int_{0}^{\infty} \frac{1}{\sigma_{k j}^{2} \sigma_{i j}^{2}} e^{-\alpha_{i k}^{j} r} d r,
\end{aligned}
$$

$$
\alpha_{i k}^{j}=1 / \sigma_{k j}^{2}+1 / \sigma_{i j}^{2} \text {. It follows that }
$$

$$
E_{i}\left\{\frac{1}{N} \sum_{k=1}^{N} \frac{1}{\pi \sigma_{k j}^{2}} e^{-\left(r / \sigma_{k j}^{2}\right)}\right\}=\frac{1}{\pi N} \sum_{k=1}^{N} \frac{1}{\sigma_{k j}^{2} \sigma_{i j}^{2} \alpha_{i k}^{j}}=\frac{1}{\pi N} \sum_{j=1}^{N} \beta_{i k}^{j},
$$

where $\beta_{i k}^{j}=1 /\left(\sigma_{i j}^{2}+\sigma_{k j}^{2}\right)$. Then, $L\left(\boldsymbol{\sigma}_{\mathbf{j}}\right)$ is calculated as follows:

$$
L\left(\boldsymbol{\sigma}_{\mathbf{j}}\right)=-\frac{1}{N} \sum_{i=1}^{N} \log _{2}\left(\frac{1}{\pi N} \sum_{k=1}^{N} \beta_{i k}^{j}\right) .
$$


Therefore, according to (18), a lower bound for $C_{\mathrm{sr}}^{j}$, which is denoted by $C_{\mathrm{sm}}^{(\mathrm{LB})}$, can be expressed as

$$
C_{\mathrm{sm}}^{(\mathrm{LB})}=-\log _{2}\left(\pi \sigma_{n}^{2} e\right)-\log _{2}\left(\frac{1}{\pi N} \sum_{k=1}^{N} \beta_{i k}^{j}\right) \leq C_{\mathrm{sr}}^{j} .
$$

2.1.2. Upper Bound of the SM Capacity. To calculate an upper bound of $C_{\mathrm{sr}}^{j}$, function $H_{i j}\left(\boldsymbol{\sigma}_{\mathbf{j}}\right)$ is defined from the integral term of (12) as

$$
H_{i j}\left(\boldsymbol{\sigma}_{\mathbf{j}}\right)=\int_{0}^{\infty} \frac{1}{\sigma_{i j}^{2}} e^{-\left(r / \sigma_{i j}^{2}\right)} \log _{2}\left(\frac{1}{N} \sum_{k=1}^{N} \frac{1}{\pi \sigma_{k j}^{2}} e^{-\left(r / \sigma_{k j}^{2}\right)}\right) d r .
$$

Using by part integral to solve, we obtain

$$
\begin{aligned}
H_{i j}\left(\boldsymbol{\sigma}_{\mathbf{j}}\right)= & -\left.e^{-\left(r / \sigma_{i j}^{2}\right)} \log _{2}\left(\frac{1}{N} \sum_{k=1}^{N} \frac{1}{\pi \sigma_{k j}^{2}} e^{-\left(r / \sigma_{k j}^{2}\right)}\right)\right|_{0} ^{\infty} \\
& -\frac{\ln (2)}{\pi N} \int_{0}^{\infty} e^{-\left(r / \sigma_{i j}^{2}\right)} \frac{\sum_{k=1}^{N}\left(1 / \sigma_{k j}^{4}\right) e^{-\left(r / \sigma_{k j}^{2}\right)}}{(1 / \pi N) \sum_{k=1}^{N}\left(1 / \sigma_{k j}^{2}\right) e^{-\left(r / \sigma_{k j}^{2}\right)}} d r,
\end{aligned}
$$

$$
\begin{aligned}
H_{i j}\left(\boldsymbol{\sigma}_{\mathbf{j}}\right)= & \log _{2}\left(\frac{1}{\pi N} \sum_{k=1}^{N} \frac{1}{\sigma_{k j}^{2}}\right) \\
& -\frac{\ln (2)}{\pi N} \int_{0}^{\infty} e^{-\left(r / \sigma_{i j}^{2}\right)} \frac{\sum_{k=1}^{N}\left(1 / \sigma_{k j}^{4}\right) e^{-\left(r / \sigma_{k j}^{2}\right)}}{\left((1 / \pi N) \sum_{k=1}^{N}\left(1 / \sigma_{k j}^{2}\right) e^{-\left(r / \sigma_{k j}^{2}\right)}\right)} d r .
\end{aligned}
$$
to 1 :

The upper bound of (22) is attained when $e^{-\left(r / \sigma_{i j}^{2}\right)}$ is equal

$$
H_{i j}\left(\boldsymbol{\sigma}_{\mathbf{j}}\right)=2 \log _{2}\left(\frac{1}{\pi N} \sum_{k=1}^{N} \frac{1}{\sigma_{k}^{2}}\right)
$$

Therefore, according to (12), an upper bound of $C_{\mathrm{sr}}^{j}$, which is denoted by $C_{\mathrm{sm}}^{(\mathrm{UB})}$, can be expressed as

$$
C_{\mathrm{sm}}^{(\mathrm{UB})}=-\log _{2}\left(\pi \sigma_{n}^{2} e\right)-2 \log _{2}\left(\frac{1}{\pi N} \sum_{k=1}^{N} \frac{1}{\sigma_{k}^{2}}\right) \geq C_{\mathrm{sr}}^{j}
$$

The lower bound and upper bound of the capacity as obtained in (19) and (24) are much easier to calculate because of their closed form. In the next section, we will show that the lower bound capacity is very close to its original capacity and can be used as a very good approximation in further analysis.

2.2. The Capacity of Relay-Destination Links. In this paper, according to the system model, the transmissions between the relays and the destination are based on a conventional communication system. For this model system, the $j$ th relay-destination capacity is obtained as

$$
C_{\mathrm{rd}}^{j}=\log _{2}\left(1+\zeta_{r, j}\right)
$$

in which $\zeta_{r, j}=\left|h_{\mathrm{rd}}^{j}\right|^{2} P_{r, j} / \sigma_{n}^{2}$, where $P_{r, j}$ is the transmission power of the $j$ th relay.

\section{Relay Selection Scheme}

Optimal relay selection, as it is expected, would improve the performance of relay networks at the expense of some computational complexity. The relay selection problem for the CSM system can be formulated as the following max-min optimization problem $(P 1)$ :

$$
P 1: \arg \max _{j}\left\{C_{\mathrm{csm}}^{j}\right\}=\arg \max _{j}\left\{\min \left\{C_{\mathrm{sr}}^{j}, C_{\mathrm{rd}}^{j}\right\}\right\} .
$$
$P 1$ are

Since $\min \left\{C_{\mathrm{sr}}^{j}, C_{\mathrm{rd}}^{j}\right\} \leq C_{\mathrm{sr}}^{j}$ or $\leq C_{\mathrm{rd}}^{j}$, two upper bounds of

$$
\left\{\begin{array}{l}
P 1(1): \arg \max _{j}\left\{C_{\mathrm{sr}}^{j}\right\} \\
P 1(2): \arg \max _{j}\left\{C_{\mathrm{rd}}^{j}\right\}
\end{array}\right.
$$

Under the assumption of equal power allocation (EPA) among the source and the relay, transmit power is given by $P_{\mathrm{s}}=P_{r, j}=\left(p_{\mathrm{tot}} / 2\right) \forall j$, and $p_{\text {tot }}$ represents the total power consumption in both transmission phases.

Firstly, $P 1(1)$ is used to derive the relay selection criterion. In the first transmission phase, because of inexistence of a closed form of the exact capacity of $\mathrm{SM}, C_{\mathrm{sm}}^{(\mathrm{LB})}$ and $C_{\mathrm{sm}}^{(\mathrm{UB})}$ are considered instead of $C_{\mathrm{sr}}^{j}$. Thus, using (26),

$$
\max _{j}\left\{C_{\mathrm{sm}}^{(\mathrm{UB})}\right\}=\max _{j}\left\{-\log _{2}\left(\pi e \sigma_{n}^{2}\right)-\frac{1}{N} \log _{2}\left(\frac{1}{\pi N} \sum_{i=1}^{N} \frac{1}{\sigma_{i j}^{2}}\right)\right\},
$$

$$
\max _{j}\left\{C_{\mathrm{sm}}^{(\mathrm{LB})}\right\}=\max _{j}\left\{-\log _{2}\left(\pi e \sigma_{n}^{2}\right)-\frac{1}{N} \sum_{i=1}^{N} \log _{2}\left(\frac{1}{\pi N} \sum_{k=1}^{N} \beta_{i k}^{j}\right)\right\} .
$$

The first term of the right-hand side of (28) is a constant value, so the entire equation can be maximized if the second term of the right-hand side of (28) has a minimum value:

$$
\begin{aligned}
\max _{j}\left\{C_{\mathrm{sm}}^{(\mathrm{UB})}\right\} & =\min _{j}\left\{\log _{2}\left(\frac{1}{\pi N} \sum_{i=1}^{N} \frac{1}{\sigma_{i j}^{2}}\right)\right\}=\min _{j}\left\{\sum_{i=1}^{N} \frac{1}{\sigma_{i j}^{2}}\right\} \\
& =\min _{j}\left\{\sum_{i=1}^{N} \frac{1}{\left|h_{\mathrm{sr}}^{i, j}\right|^{2}+\left(\sigma_{n}^{2} / P_{\mathrm{s}}\right)}\right\} \cong \min _{j}\left\{\sum_{i=1}^{N} \frac{1}{\left|h_{\mathrm{sr}}^{i j}\right|^{2}}\right\} .
\end{aligned}
$$


For the same reason,

$$
\begin{aligned}
\max _{j}\left\{C_{\mathrm{sm}}^{(\mathrm{LB})}\right\} & =\min _{j}\left\{\sum_{i=1}^{N} \log _{2}\left(\sum_{k=1}^{N} \beta_{i k}^{j}\right)\right\} \\
& =\min _{j}\left\{\sum_{i=1}^{N} \log _{2}\left(\sum_{k=1}^{N} \frac{1}{\sigma_{k j}^{2}}+\frac{1}{\sigma_{i j}^{2}}\right)\right\} \\
& =\min _{j}\left\{\sum_{i=1}^{N} \log _{2}\left(\frac{N}{\sigma_{i j}^{2}}+\sum_{k=1}^{N} \frac{1}{\sigma_{k j}^{2}}\right)\right\} \min _{j}\left\{\sum_{i=1}^{N} \frac{1}{\sigma_{i j}^{2}}\right\} \\
& \cong \min _{j}\left\{\sum_{i=1}^{N} \frac{1}{\left|h_{\mathrm{sr}}^{i j}\right|^{2}}\right\} .
\end{aligned}
$$

The term $\min _{j}\left\{\sum_{i=1}^{N}\left(1 /\left|h_{s r}^{i j}\right|^{2}\right)\right\}$ is repeated for the upper and lower bounds and could be used as a relay selection criterion (RSC):

$$
\operatorname{RSC} 1=\underset{j}{\operatorname{argmin}}\left\{\sum_{i=1}^{N} \frac{1}{\left|h_{\mathrm{sr}}^{i j}\right|^{2}}\right\} .
$$

Additionally, according to [14], criterion RSC2 = $\underset{j}{\operatorname{argmin}}\left\{1 /\left|h_{r d}^{j}\right|\right\}$ is chosen for relay selection, and this could be used as the solution of $P 1(2)$. Relay selection that is based on RSC1 or RSC2 might not yield the optimal solution. Here, we propose a new method that is based on the properties of max-min optimization for solving $P 1$. The following lemma is instrumental for obtaining the optimum solution based on the link quality in both transmission phases.

Lemma 1. If $j^{*}=\underset{j}{\operatorname{argmax}}\left\{C_{s r}^{j}\right\}$ and $C_{s r}^{j^{*}}=\min \left\{C_{s r}^{j^{*}}, C_{r d}^{j^{*}}\right\}$, where $j^{*}$ is the index of selected relay, then $j^{*}=\operatorname{argmax}$ $\left\{\min \left\{C_{s r}^{j}, C_{r d}^{j}\right\}\right\}$.

Proof. From $\min \left\{C_{\mathrm{sr}}^{j}, C_{\mathrm{rd}}^{j}\right\} \leq C_{\mathrm{sr}}^{j}$, it follows that

$$
\max _{j}\left\{\min \left\{C_{\mathrm{sr}}^{j}, C_{\mathrm{rd}}^{j}\right\}\right\} \leq \max _{j}\left\{C_{\mathrm{sr}}^{j}\right\}
$$

First, we define $j^{*}=\operatorname{argmax}\left\{C_{\mathrm{sr}}^{j}\right\}$. From (33), we deduce that the equality holds if $C_{\mathrm{sr}}^{j^{*}}=\min \left\{C_{\mathrm{sr}}^{j^{*}}, C_{\mathrm{rd}}^{j^{*}}\right\}$. On the other hand, when the inequality is fulfilled with equality in (33), the optimal solution is obtained, namely, $\max _{j}\left\{\min \left\{C_{\mathrm{sr}}^{j}\right.\right.$, $\left.\left.C_{\mathrm{rd}}^{j}\right\}\right\}=\max _{j}\left\{C_{\mathrm{sr}}^{j}\right\}$. Then, the solution can be expressed as $j^{*}=\underset{j}{\operatorname{argmax}}\left\{\min \left\{C_{\mathrm{sr}}^{j}, C_{\mathrm{rd}}^{j}\right\}\right\}$.

In the proposed method, namely, the optimum relay selection algorithm (ORSA), first, we sort the relays according to RSC1. Then, a relay with the highest priority is selected. If the chosen relay satisfies the conditions of Lemma 1, it is the optimal relay; otherwise, another relay with the next top priority is selected. In the case of the multiple relay selection scheme, this process will continue until the required number of relays have been selected. Computing $C_{\mathrm{sm}}^{(\mathrm{LB})}$ for the large numbers of relays, $(K \gg 1)$, is much faster; therefore, we can consider $C_{\mathrm{sm}}^{(\mathrm{LB})}$ instead of $C_{\mathrm{csm}}^{j}$ in practical applications. Using an exhaustive search to acquire optimum selection has exponential complexity of $O(K) O$ $\left(\operatorname{Fun}\left(C_{1, j}\right)+\operatorname{Fun}\left(C_{2, j}\right)\right)$, in which $O(\operatorname{Fun}(\cdot))$ is the computational complexity of any function. However, we propose a heuristic selection algorithm with rational complexity. In the next section, we will see that this method has much less computational complexity compared to the exhaustive search algorithm.

In comparison with exhaustive search, the proposed selection algorithm has some features, which can be noticeable and practical due to its complexity, which is $O$ (Fun $(\mathrm{RSC} 1)+\mathrm{Fun}(\mathrm{RSC} 2))$ in the best situation and $O(K) O($ Fun $(\mathrm{RSC} 1)+\mathrm{Fun}(\mathrm{RSC} 2))$ in the worst one.

The two aforementioned criteria, namely, RSC1 and RSC2, consider the link quality in only one of the transmission phases. The maximum harmonic mean (MHM) relay selection criterion is an important criterion in relay selection, as it considers the channel qualities of both transmission phases. Using SM in the first transmission phase enforces a change in the relay selection criterion as it is named modified maximum harmonic mean (MMHM) and given by

$$
\mathrm{MMHM}=\arg \max _{j}\left\{\frac{\overline{\left|h_{\mathrm{SR}}\right|^{2}}\left|h_{\mathrm{rd}}^{j}\right|^{2}}{\overline{\left|h_{\mathrm{SR}}\right|^{2}}+\left|h_{\mathrm{rd}}^{j}\right|^{2}}\right\},
$$

where $\overline{\left|h_{\mathrm{SR}}\right|^{2}}$ is interpreted as the mean effective channel gain of SRL and is expressed as follows:

$$
\overline{\left|h_{\mathrm{SR}}\right|^{2}}=\left(\frac{1}{N} \sum_{i=1}^{N} \frac{1}{\left|h_{\mathrm{sr}}^{i j}\right|^{2}}\right)^{-1} .
$$

In addition to the previously defined criteria, we present MMHM in CSM as a new selection criterion for benchmarking ORSA.

\section{Simulation Results}

This section presents a simulation study and evaluation of the CSM system model (Figure 1) in terms of channel capacity. The study also evaluates the earlier presented relay selection criteria.

First, the original and the lower and upper bounds of the SM system as derived in Section 2 are calculated under the assumption of 8 antennas at the source. Figure 2 shows that the lower bound of the SM capacity is very close to its original function. Therefore, due to the nonclosed form and the computational complexity of the original function for the SM 


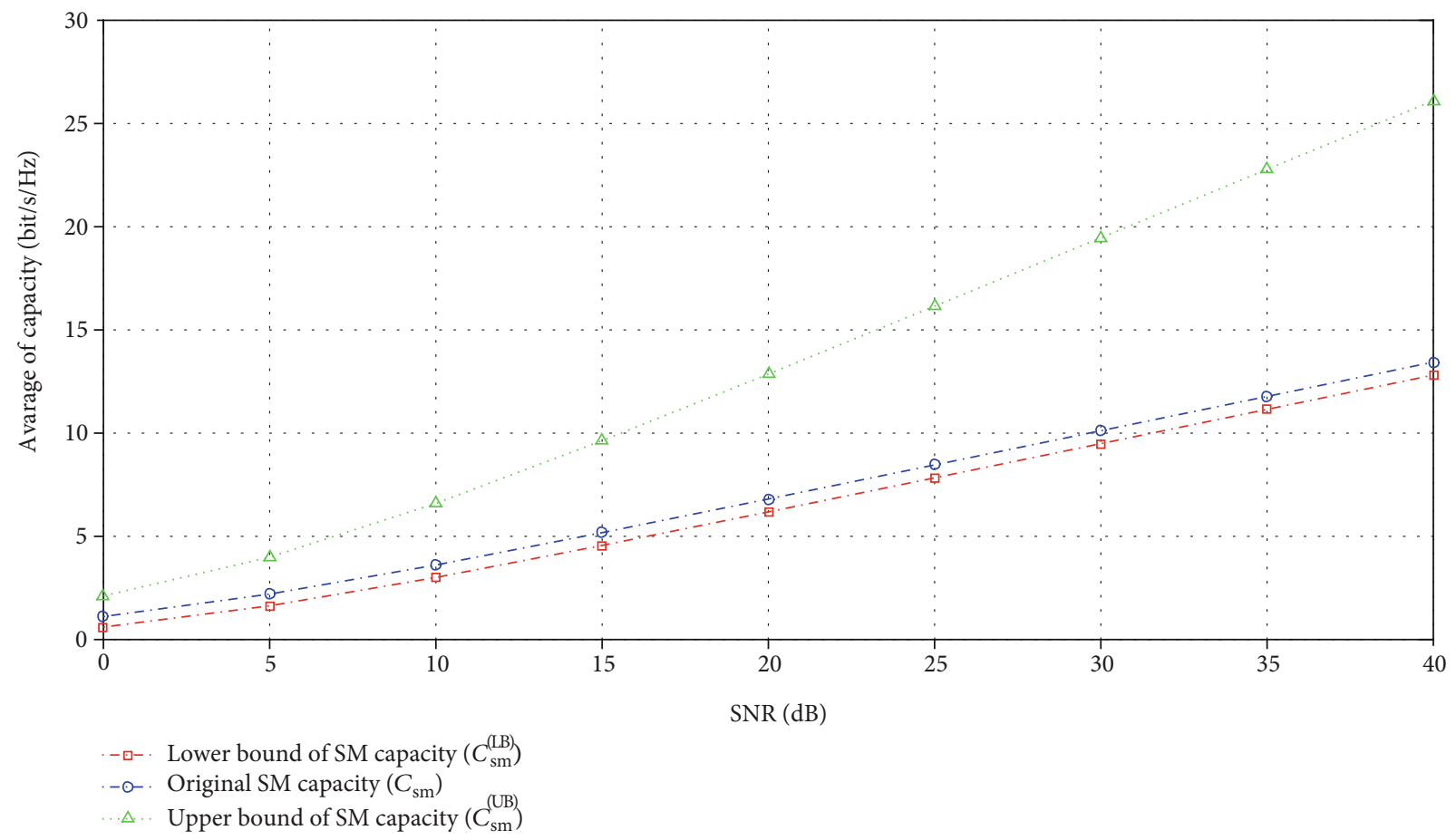

FIGURE 2: SM capacity versus SNR in the system model.
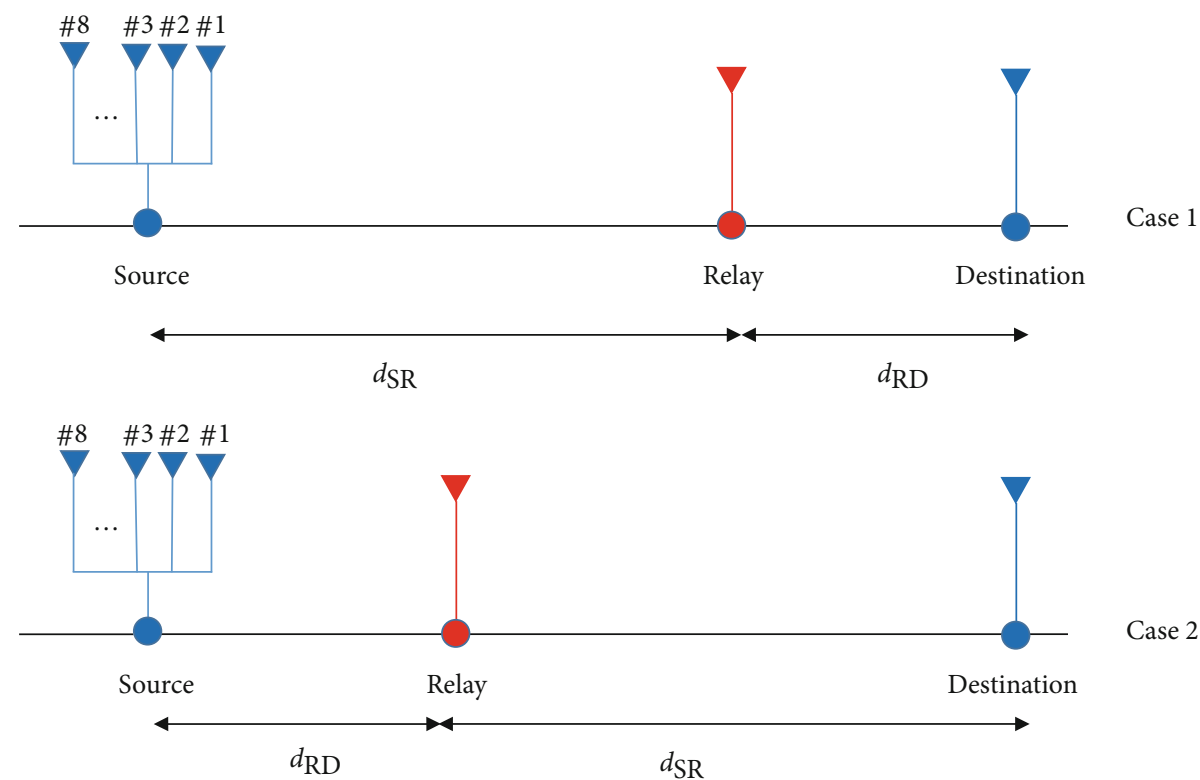

Case 2

FIGURE 3: Simulation case studies.

capacity, we can use the lower bound as a closed form approximation for the capacity analysis in this study.

Next, a numerical simulation based on the proposed relay selections RSC1, RSC2, MMHM, and ORSA is conducted. Also, the random relay selection (RRS) scheme is used to benchmark the results. Based on the locations of the source, relays, and destination, we will evaluate the criteria for the relay selection in two case studies, as illustrated in Figure 3.

In the simulation study, we assumed that the number of transmitted antennas is $N=8$, and BPSK modulation is applied, i.e., $M=2$. A single relay would be selected among the available eight terminals. Equal power allocation is considered where $p_{\mathrm{s}}=p_{\mathrm{r}}=p_{\text {tot }} / 2$.

In the first case study, the relay is closer to the destination than the source, i.e., $d_{\mathrm{SR}}>d_{\mathrm{RD}}$ (Figure3). The results in Figure 4 show that RSC1 outperforms both RSC2 and RRS since it considers the quality of the first transmission link as a selection criterion, and according to (1), this link has a stronger effect on the system capacity. Figure 5 shows that for a similar reason, in the second case study where the relay 


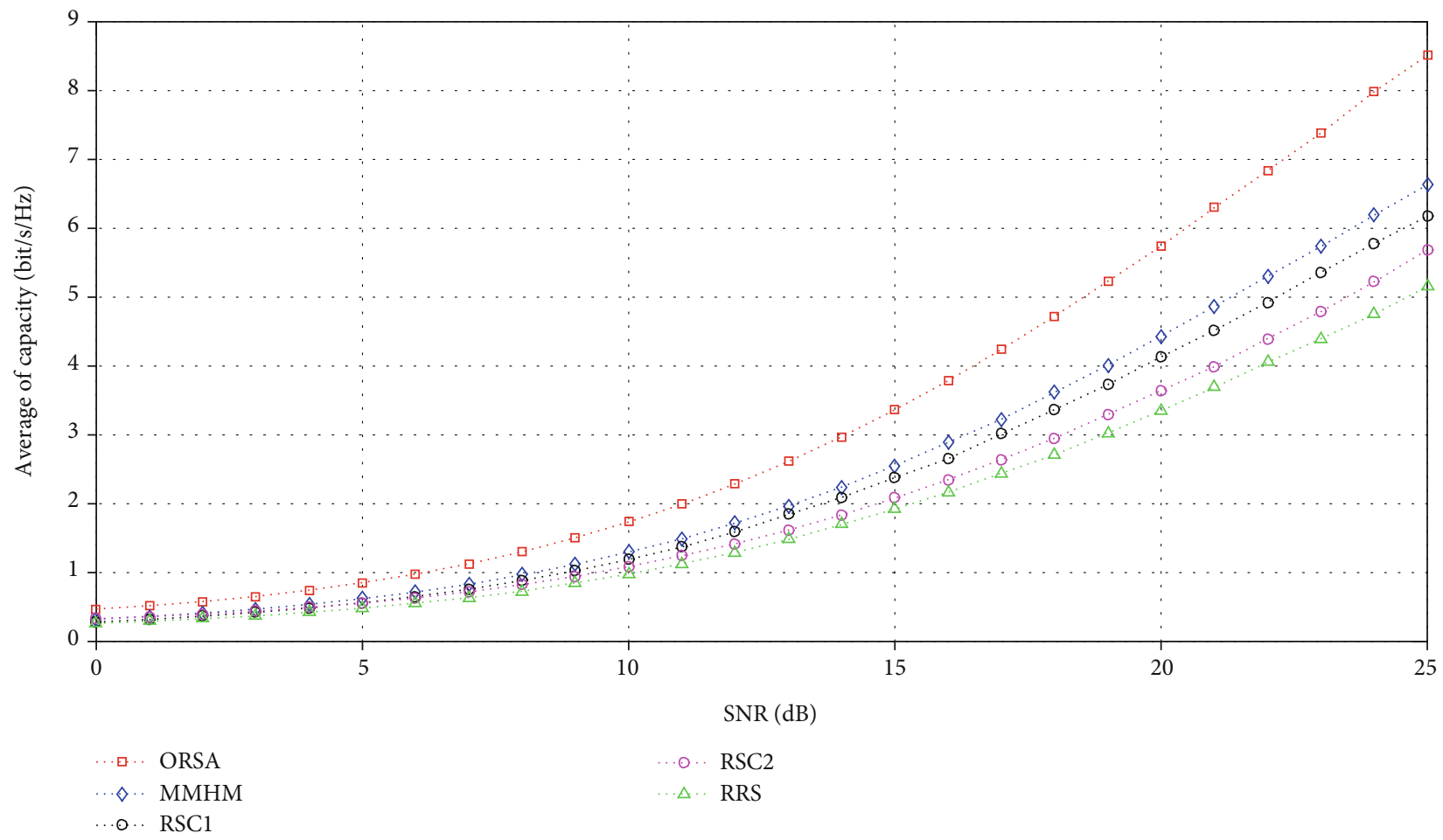

FIgURE 4: The capacity of CSM network using different selection criteria in case $1\left(\mathbf{d}_{\mathbf{S R}}>\mathbf{d}_{\mathrm{RD}}\right)$.

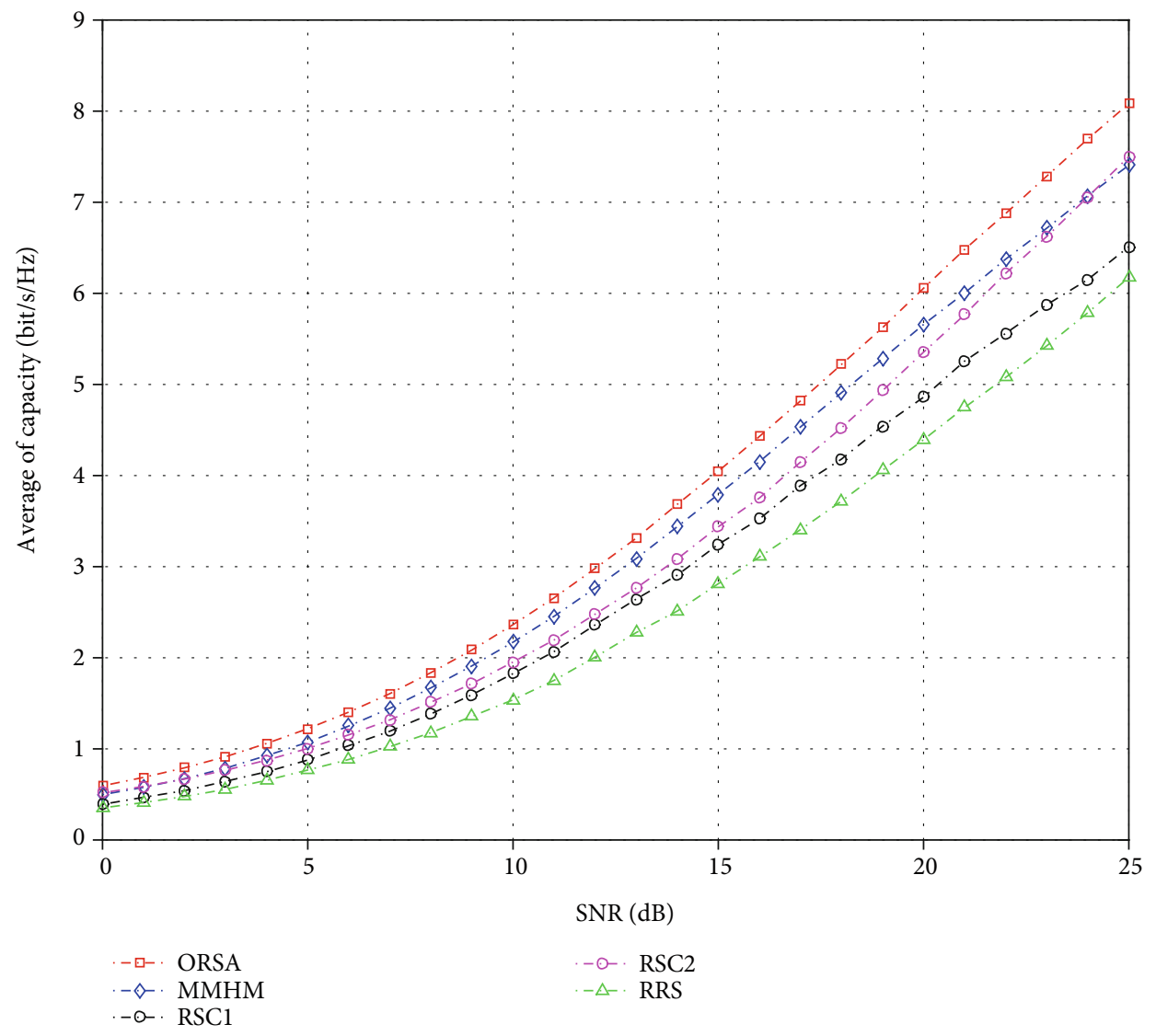

FIGURE 5: Capacity of CSM network for different selection criteria in case $2\left(\mathbf{d}_{\mathbf{S R}}<\mathbf{d}_{\mathbf{R D}}\right)$. 


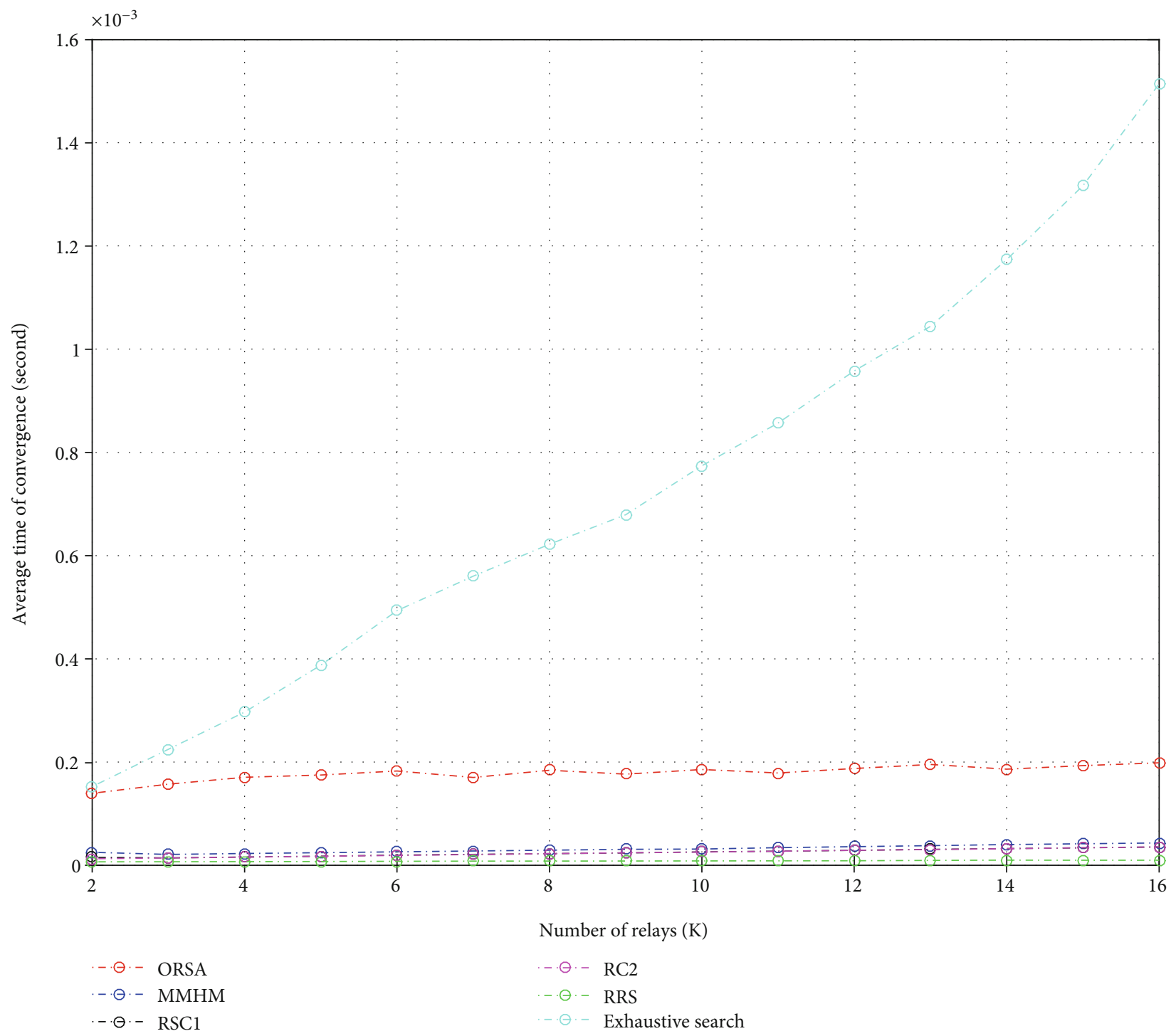

FIGURE 6: Comparison among relay selection methods in terms of computational complexity.

is closer to the source $\left(d_{\mathrm{SR}}<d_{\mathrm{RD}}\right)$ in contrast to the earlier case, RSC2 outperforms RSC1. In the low SNR regime (0$10 \mathrm{~dB}$ ), the channel noise generally dominates the impairments; however, in the high-SNR regime, the difference in the variances affects the performances of the methods.

For both cases, MMHM portrays its efficacy as a selection policy where it outperforms RSC1 and RSC2. It considers the quality of the links in both transmission phases regardless of the position of the relay. However, according to the results in Figures 4 and 5, the ORSA algorithm behaves better than the other methods. Similarly to MMHM, it considers both transmission links to define an optimal solution of the $P 1$ problem and identify the optimum relay to forward the information signal. At the expense of computational overhead, all the suggested selection policies record higher performance than no selection policy where a relay is selected randomly regardless of its channel coefficients or its position.

To reflect the computational overhead that each selection policy would enforce, the average time for the computational operations in the proposed methods for single relay selection versus the number of the available relays is presented in Figure 6. The proposed optimal solution for $P 1$ that is based on ORSA is far less complicated compared to the exhaustive search, which has linear computational complexity with respect to the number of relays. Therefore, ORSA is a highly effective algorithm for realizing optimal performance in CSM with relay selections. Moreover, MMHM, RSC1, and RSC2 record lower complexity than ORSA since it consumes less time to select a relay at the expense of lower performance.

\section{Conclusions}

A relay-aided CSM network is studied to increase the coverage area and leverage the channel capacity. Theoretical analysis of the channel utilization in the CSM network is presented, and the lower and upper bounds of the capacity is derived. The lower bound capacity function is exploited in the optimal relay selection problem because of its 
simplicity and its much-approximated values to the original SM capacity. Different relay selection methods and criteria with different computational complexity are applied in the system to increase the transmission capacity. The methods and the criteria are evaluated in terms of the channel capacity and computational time. All the suggested criteria behave better than no selection policy at the expense of a cost.

It has been found that ORSA has lower computational complexity than the exhaustive search and higher performance than the other suggested selection policies. MMHM is presented as another important selection criterion to be used in CSM, which is originally based on MHM that is widely applied in conventional cooperative networks. The criterion also yields a suboptimal solution in terms of network performance and an equivalent complexity to that for RSC1 and RSC2.

\section{Data Availability}

The data used to support the findings of this study are available from the corresponding author upon request.

\section{Conflicts of Interest}

The authors declare that they have no conflicts of interest.

\section{References}

[1] P. Yang, M. Di Renzo, Y. Xiao, S. Li, and L. Hanzo, "Design guidelines for spatial modulation," IEEE Communications Surveys \& Tutorials, vol. 17, no. 1, pp. 6-26, 2015.

[2] M. Wen, B. Zheng, K. J. Kim et al., "A survey on spatial modulation in emerging wireless systems: research progresses and applications," IEEE Journal on Selected Areas in Communications, vol. 37, no. 9, pp. 1949-1972, 2019.

[3] D. Gunduz, M. A. Khojastepour, A. Goldsmith, and H. V. Poor, "Multi-hop MIMO relay networks: diversitymultiplexing trade-off analysis," IEEE Transactions on Wireless Communications, vol. 9, no. 5, pp. 1738-1747, 2010.

[4] J. Mietzner, R. Schober, L. Lampe, W. Gerstacker, and P. Hoeher, "Multiple-antenna techniques for wireless communications - a comprehensive literature survey," IEEE Communications Surveys \& Tutorials, vol. 11, no. 2, pp. 87-105, 2009.

[5] J. Jeganathan, A. Ghrayeb, and L. Szczecinski, "Spatial modulation: optimal detection and performance analysis," IEEE Communications Letters, vol. 12, no. 8, pp. 545-547, 2008.

[6] Y. Yang and B. Jiao, "Information-guided channel-hopping for high data rate wireless communication," IEEE Communications Letters, vol. 12, no. 4, pp. 225-227, 2008.

[7] C. Liu, M. Ma, Y. Yang, and B. Jiao, "Optimal spatial-domain design for spatial modulation capacity maximization," IEEE Communications Letters, vol. 20, no. 6, pp. 1092-1095, 2016.

[8] T. L. Narasimhan and A. Chockalingam, "On the capacity and performance of generalized spatial modulation," IEEE Communications Letters, vol. 20, no. 2, pp. 252-255, 2016.

[9] H. Bitra and P. Ponnusamy, "Closed form capacity expression of spatial modulation using hypergeometric series," in 2020 International Conference on Computation, Automation and Knowledge Management (ICCAKM), pp. 527-530, Dubai, United Arab Emirates, January 2020.
[10] K. Zarifi, M. Abuthinien, A. Ghrayeb, and S. Affes, "Relay selection schemes for uniformly distributed wireless sensor networks," in 2009 IEEE Wireless Communications and Networking Conference, pp. 1-6, Budapest, Hungary, April 2009.

[11] X. Chen, T. Siu, Q. F. Zhou, and F. C. M. Lau, "High-SNR analysis of opportunistic relaying based on the maximum harmonic mean selection criterion," IEEE Signal Processing Letters, vol. 17, no. 8, pp. 719-722, 2010.

[12] W. Xia, S. Shao, and J. Sun, "Relay selection strategy for device to device communication," in 2009 IEEE Wireless Communications and Networking Conference, pp. 318-323, Beijing, China, April 2013.

[13] J. Crawford and Y. Ko, "Cooperative OFDM-IM relay networks with partial relay selection under imperfect CSI," IEEE Transactions on Vehicular Technology, vol. 67, no. 10, pp. 9362-9369, 2018.

[14] J. A. Hassan and S. A. Mustafa, "An efficient partner selection method to overcome the interference effect in wireless networks," IEEE Access, vol. 7, pp. 33677-33685, 2019.

[15] R. Mesleh and S. S. Ikki, "Performance analysis of spatial modulation with multiple decode and forward relays," IEEE Wireless Communications Letters, vol. 2, no. 4, pp. 423-426, 2013.

[16] S. R. Hussain, S. Shakeera, and K. R. Naidu, "BER analysis of amplify and forward scheme with best relay selection in space shift keying system," in 2015 International Conference on Communications and Signal Processing (ICCSP), pp. 17221726, Melmaruvathur, India, April 2015.

[17] S. Narayanan, H. Ahmadi, and M. F. Flanagan, "On the performance of spatial modulation MIMO for full-duplex relay networks," IEEE Transactions on Wireless Communications, vol. 16, no. 6, pp. 3727-3746, 2017.

[18] G. Altın, Ü. Aygölü, E. Basar, and M. E. Çelebi, "Multipleinput-multiple-output cooperative spatial modulation systems," IET Communications, vol. 11, no. 15, pp. 2289-2296, 2017.

[19] M. S. Muhareb, A. M. Abu-Hudrouss, and M. O. E. Astal, "Quadrature spatial modulation for wireless relaying networks," in 2018 International Conference on Innovative Trends in Computer Engineering (ITCE), pp. 166-169, Aswan, Egypt, Febuary 2018.

[20] K. Pang, Z. Bai, P. Ma et al., "Design and analysis of quadrature spatial modulation with multiple decode-and-forward relays," in 2019 19th International Symposium on Communications and Information Technologies (ISCIT), pp. 339-343, Ho Chi Minh City, Vietnam, September 2019.

[21] O. M. Kandelusy and S. M. H. Andargoli, "Outage performance of decode-and-forward (DF)-based multiuser spectrum sharing relay system with direct link in the presence of primary users' power," IET Communications, vol. 12, no. 3, pp. 246254, 2018.

[22] F. A. Prisecaru and H. Hass, Mutual Information and Capacity of Spatial Modulation Systems, School of Engineering and Science Jacobs University, 2007. 